\title{
Erratum: Helicobacter pylori adhesin HopQ engages in a virulence-enhancing interaction with human CEACAMs
}

Anahita Javaheri, Tobias Kruse, Kristof Moonens, Raquel Mejías-Luque, Ayla Debraekeleer, Carmen I. Asche, Nicole Tegtmeyer, Behnam Kalali, Nina C. Bach, Stephan A. Sieber, Darryl J. Hill, Verena Königer, Christof R. Hauck, Roman Moskalenko, Rainer Haas, Dirk H. Busch, Esther Klaile, Hortense Slevogt, Alexej Schmidt, Steffen Backert, Han Remaut, Bernhard B. Singer and Markus Gerhard

Nature Microbiology 2, 16189 (2016); published 17 October 2016; corrected 7 November 2016.

The original version of this Article contained incorrect labels in Figs $3 \mathrm{~b}$ and 4e. In Fig. 3b, the second western blot panel should have been labelled 'CEACAM1'. In Fig. 4e, the final two values on the y axis should have read 'I91A' and 'I91T'. All versions of the Article have been updated. We apologize for any confusion this may have caused. 